\title{
E-mail Addresses of IAU Colloquium Attendees
}

Abada-Simon, Meil

Aigner, Tobias

Andronov, Ivan L.

Ball, Bill

Benz, Arnold O.

Beskin, Grigory M.

Beskrovnaya, Nina

Bowyer, Stuart

Brandner, Wolfgang

Bruch, Albert

Byrne, P. Brendan

Casperson, Donald E.

Castro-Tirado, Alberto

Csepura, György

Dravins, Dainis

Duerbeck, Hilmar

Fendt, Christian

Fishman, Gerald J.

Franciosini, Elena

Gahm, Gösta

Gershberg, Roald

Glukhov, Alexander

Götz, Woldemar

Greiner, Jochen

Gullbring, Erik

Hartmann, Dieter

Hazen, Martha L.

Hildebrandt, Joachim

Hojaev, Alisher

Hudec, Rene

Ikhsanov, Nazar

Karetnikov, Valentin G.

Kato, Mariko abada@fys.ruu.nl

tba@mpe-garching.mpg.de

root@astro.odessa.ua

wnb@sa1.star.uclan.ac.uk

benz@astro.phys.ethz.ch

beskin@sao.stavropol.su

beskr@gaoran.spb.su

bowyer@cea.berkeley.edu

wolfgang@astro.uni-wuerzburg.de

albert@cygnus.uni-muenster.de

pbb@star.arm.ac.uk

dcasperson@lanl.gov

ajct@laeff.esa.es

csepura@tigris.klte.hu

dainis@astro.lu.se

hilmar@cygnus.uni-muenster.de

fishman@ssl.msfc.nasa.gov

francio@arcetri.astro.it

gahm@emil.astro.su.se

gershberg@crao.crimea.ua

cosmos@physfac.rostov-na-donu.su

jcg@mpe-garching.mpg.de

erik@jarlabanke.astro.su.se

hartmann@biophy.phys.clemson.edu

martha@cfa.harvard.edu

jhildebrandt@aip.de

root@ipmce.tashkent.su

rhudec@asu.cas.cz

nri@pulkovo.spb.su

root@astro.odessa.ua

mariko@educ.cc.keio.ac.jp 
Katsova, Maria M.

Klapdor-Kleingrothaus, H.V.

Klis, Michiel van der

Konstantinova-Antova, Renada

Kouveliotou, Chryssa

Krivosheina, Irina

Kroll, Peter

Krüger, Albrecht

Kürster, Martin

Kurtanidze, Omar M.

laDous, Constanze

Lapidus, Iosif

Larsson, Stefan

Lehmann, Thomas

Livshits, Moissei

Lührs, S.

Luthardt, Elena

Luthardt, Rainer

Mahmoud, Farouk M.

Markina, Ala

Meinunger, Isolde

Merck, Martin

Minarini, Roberto

Mirabel, Felix

Mirzoyan, Ludwig V.

Moskalenko, Evgeni

Neizvestny, Sergei

Notni, Peter

Oleak, Hans

Ottmann, Renate

Özkan, Türker

Paciesas, William S.

Pagano, Isabella

Pallavicini, Roberto

Pan, Hongchao

Panferov, Alexander

Paradijs, Jan van

Poveda, Arcadio Ricalde

Preibisch, Thomas

Priest, Eric R.

Pustil'nik, Lev

Pustyl'nik, Izold

Reimer, Olaf

Richter, Gerold A.

Ricker, George maria@sai.msk.su

michiel@astro.uva.nl

belogr\%bgearn.bitnet@vm.gmd.de

kouveliotou@batse.msfc.nasa.gov

kroll@nova.tat.physik.uni-tuebingen.de

akrueger@aip.de

mak@mpe-garching.mpg.de

gmelik@dtapha.kheta.georgia.su

cld@vilspa.esa.es

lapidus@mail.ast.cam.ac.uk

stefan@astro.su.se

tlehmann@eso.org

root@astro.odessa.ua

root@astro.odessa.ua

mnm@mpe-garching.mpg.de

minarini@sao.stavropol.su

33590::MIRABEL

tarkh@acsci.armenia.su

moskal@sai.msk.su

pnotni@aip.de

holeak@aip.de

reo@mpe.mpe-garching.mpg.de

ik001@triuvm11.bitnet

paciesas@fender.msfc.nasa.gov

isabella@peroni.ct.astro.it

pallavic@arcetri.astro.it

hpan@thphys.ox.ac.uk

jvp@astro.uva.nl

poveda@redvax1.dgsca.unam.mx

preib@astro.uni-wuerzburg.de

eric@dcs.st-andrews.ac.uk

lev@yarden.yarden.ac.il

izold@jupiter.aai.ee

reimer@sicip1.physik.uni-siegen.de

grr@space.mit.edu 
Roizman, Genady

Rößiger, Siegfried

Samus, Nikolai

Sanwal, B.B.

Schwarz, Robert

Seitter, Waltraut

Shakhovskaya, Nadezhda I.

Shapiro, Maurice

Sokolov, Vladimir V.

Stegert, Jörg

Stepanov, Alexander V.

Sunyaev, Rashid

Terekhov, Oleg

Torricelli, Guinetta

Trigilio, Corrado

Tsvetkov, Milcho

Tsvetkova, Katya

Umana, Grazia

Vanderspek, Roland

Ventura, Rita

Verlyuk, Irina A.

Vogt, Nikolaus

Wang, Jun-jie

Welsh, William F.

Winterberg, Juergen

Yudin, Ruslan

Zhilyaev, Boris E.

Zinnecker, Hans lev@yarden.yarden.ac.il

samus@sai.msu.su

rschwarz@aip.de

waltraut@cygnus.uni-muenster.de

nish@crao.crimea.ua

sokolov@sao.stavropol.su

jstegert@astro.uni-bonn.de

stepanov@rt22.crimea.ua

sunyaev@]

oterekho@esoc1.bitnet

gtorricelli@arcetri.astro.it

ctrigilio@astrct.ct.astro.it

tsvetkov@bgearn.bitnet

tsvetkova@bgearn.bitnet

gumana@ct.astro.it

roland@blitz.mit.edu

rventura@astrct.ct.astro.it

maouas@gluk.apc.org

vogt@tat.physik.uni-tuebingen.de

yac@bao01.bao.ac.cn

wfw@astro.keele.ac.uk

juergen@cygnus.uni-muenster.de

ruslan@gaoran.spb.su

maouas@gluk.apc.org

hans@astro.uni-wuerzburg.de 